\title{
Flutuação populacional de gafanhotos na Floresta Nacional de Chapecó, Santa Catarina
}

\author{
Grasshopper Population fluctuation in the National Forest of Chapeco, Santa Catarina
}

\author{
Cladis Juliana Lutinski ${ }^{{ }^{*}}$ Flávio Roberto Mello Garcia' ${ }^{\mathrm{I}}$ Maria Kátia Matiotti da Costa ${ }^{\mathrm{II}}$ \\ Junir Antonio Lutinski ${ }^{\mathrm{I}}$
}

\begin{abstract}
Com o objetivo de verificar a flutuação populacional de gafanhotos na Floresta Nacional de Chapecó (FLONA), em Santa Catarina, foram realizadas coletas semanais durante o período de dezembro de 2003 a dezembro de 2004 com armadilhas do tipo pit-fall, rede de varredura, guarda-chuva entomológico e malaise. Os picos populacionais para a maioria das espécies ocorreram durante os meses de dezembro, janeiro e fevereiro. A análise de regressão (5\%) apontou relação significativa entre os fatores climáticos e os níveis populacionais de Allotruxalis gracilis (Giglio-Tos, 1897), Parorphula graminea Bruner, 1900, Rhammatocerus brunneri (Giglio - Tos, 1895) e Xyleus discoideus discoideus (Serville, 1831).
\end{abstract}

Palavras-chave: níveis populacionais, Orthoptera, Acridoidea, Tetrigoidea, Tridactyloidea.

\section{ABSTRACT}

In order to verify the grasshopper population dynamics in the National Forest of Chapecó, SC., weekly collections were made between December 2003 and December 2004, using pit-fall traps, sweep nets, entomological umbrellas and malaises traps. The population peaks for most of the species was reached in December, January and February. The regression analysis (5\%) showed significant relation between the climatic factors and the population levels of Allotruxalis gracilis (Giglio-Tos, 1897), Parorphula graminea Bruner, 1900, Rhammatocerus brunneri (Giglio - Tos, 1895) and Xyleus discoideus discoideus (Serville, 1831).

Key words: population levels, Orthoptera, Acridoidea, Tetrigoidea, Tridactyloidea.
Com o emergente interesse pelas questões ambientais, tornou-se evidente a necessidade de um conhecimento mais amplo sobre a biodiversidade, bem como a biologia e a ecologia das espécies (LUTINSKI \& GARCIA, 2005). Os ortópteros datam, nos registros fósseis, de aproximadamente 300 milhões de anos, e atualmente são registradas cerca de 20.000 espécies, distribuídas em 28 famílias (MEYER, 2005). Eles são conhecidos mundialmente e tem importância econômica relevante devido aos danos econômicos causados em áreas agrícolas por algumas espécies (COSTA-NETO, 2000).

As populações de gafanhotos flutuam com o passar do tempo, algumas alcançam dispersão global, enquanto outras alcançam dispersão regional, porém, a maioria permanece com baixas densidades. A flutuação geralmente está associada com as características ambientais (RICHMAN et al., 1993). Temperatura e umidade são as variáveis principais para a sobrevivência das populações de gafanhotos, afetando o desenvolvimento e a sobrevivência das formas jovens (ROSALES, 1996). Outro fator que interfere no desenvolvimento é o nível de precipitação, que afeta a alimentação (RICHMAN et al., 1993). Portanto, a temperaturas, a umidade e a precipitação em situações adversas interferem na flutuação das populações. Portanto, este trabalho teve como objetivo verificar a flutuação populacional de gafanhotos na

\footnotetext{
IPrograma de Mestrado em Ciências Ambientais, Universidade Comunitária Regional de Chapecó (UNOCHAPECÓ), Laboratório de Entomologia, CP 747, 89809-000, Chapecó, SC, Brasil. E-mail: lutinski@unochapeco.edu.br. *Autor para correspondência. IIFaculdade de Biociências/Laboratório Entomologia, Porto Alegre, RS, Brasil.
} 
Floresta Nacional de Chapecó (FLONA), em Santa Catarina (SC), observando a interferência dos fatores ecológicos (temperatura, umidade e precipitação) sobre o grupo.

O estudo foi conduzido na Gleba I da FLONA, localizada no município de Guatambú, SC, na localidade da Fazenda Zandavalli (2705' 50”'S; 52 46' $40^{\prime}$ W), em três áreas, com cerca de 5ha cada uma. A área 1 tem como vegetação predominante uma plantação de eucalipto (Eucalyptus saligna Smith, 1797 e Eucalyptus grandis Hill, 1862) com três anos de idade. A área 2 é coberta por mata com araucária (Floresta Ombrófila Mista) e Floresta Estacional Decidual. Aárea 3 está coberta por uma plantação de pinus (Pinus taeda Linnaeus 1753 e Pinus elliottii Engelm, 1880) com 45 anos de idade (IBAMA, 2003).

As amostragens foram semanais no período de dezembro de 2003 a dezembro de 2004. Nas coletas, foram utilizadas armadilhas do tipo ativo (rede entomológica e guarda-chuva entomológico) e armadilhas passivas (pit-fall e malaise). O esforço amostral empregado por coleta, igualmente em cada área, consistiu: em 20 minutos de varredura para rede entomológica; em aproximadamente 60 sacudidelas em um total de 10 arbustos para o guarda-chuva entomológico; em transectos lineares da borda para o interior com 10 armadilhas em intervalos de 10 metros entre si para pit-fall e uma armadilha do tipo malaise instalada na região central de cada área.

Os espécimes foram acondicionados em álcool $70 \%$ e transportados ao laboratório de Entomologia da Universidade Comunitária Regional de Chapecó (UNOCHAPECÓ), onde foram triados e identificados. A confirmação dos táxons foi realizada pela Prof $\stackrel{\text { a }}{\text {. Dra }}$. Maria Kátia Matiotti da Costa, do Departamento de Pós-Graduação em Biociências, da Pontifícia Universidade Católica do Rio Grande do Sul, com base na classificação proposta por ROWELL (2001).

Para a avaliação da influência dos fatores ecológicos sobre as espécies, foram feitos cálculos de regressão múltipla em função do número total de insetos de cada espécie com os elementos meteorológicos temperatura máxima, temperatura média, temperatura mínima, umidade máxima, umidade média, umidade mínima e precipitação mensal, fornecidos pela Empresa de Pesquisa Agropecuária e Extensão Rural de Santa Catarina S.A (EPAGRI/CLIMERH) . Para a análise estatística, foi adotada a significância de 5\%, sendo utilizado o software STATISTICA 6.0.
Foram coletados 2.325 gafanhotos, somando 25 espécies: Família Acrididae: Abracris flavolineata (De Geer, 1773), Aleuas lineatus Stål, 1878, Allotruxalis gracilis (Giglio-Tos, 1897), Amblytropidia sp., Cylindrotettix chacoensis Roberts, 1975, Cylindrotettix sp., Dichroplus elongatus (Giglio-Tos, 1894), Dichroplus misionensis Carbonell, 1968, Metaleptea adspersa (Rehn, 1944), Orphulella punctata (De Geer, 1773), Parorphula graminea Bruner, 1900, Peruvia nigromarginata (Scudder, 1875), Rhammatocerus brunneri (Giglio - Tos, 1895), Rhammatocerus pictus (Bruner, 1900), Ronderosia bergi (Stål, 1878), Scotussa lemniscata (Stål, 1861) e Staurorhectus longicornis longicornis Giglio-Tos, 1897. Família Ommexechidae: Ommexecha virens (Servile, 1831). Família Romaleidae: Chromacris speciosa (Thumberg, 1824), Staleochlora viridicata viridicata (Serville, 1839), Xyleus discoideus discoideus (Serville, 1831), Zoniopoda iheringi Pictet \& Saussure, 1887 e Zoniopoda tarsata (Serville, 1831). Família Tetrigidae: Tetrix subulata Linaeus, 1761. Família Tridactylidae: Tridactylus politus Bruner, 1916. A proporção de cada espécie coletada encontra-se na tabela 1. A maior abundância foi registrada de outubro a maio, com picos populacionais de dezembro a fevereiro, ocasião dos maiores índices de temperatura na região (Tabela 2), declinando a abundância durante a passagem dos meses mais frios (Tabela 1) Esse resultado corrobora os resultados do estudo de ROSALES (1996), que identificou que as temperaturas acima de $16^{\circ} \mathrm{C}$ favorecem a presença de gafanhotos.

As espécies A. gracilis, D. misionensis e $\boldsymbol{R}$. bergi destacaram-se com maiores índices de incidência. Estas espécies são potencialmente pragas e generalistas de ambientes (CARBONELL et al., 2006; DEBREY et al., 1993). O resultado encontrado corrobora o resultado obtido pela EMBRAPA (1996), em estudos com a espécie Rhammatocerus schistocercoides (Rehn, 1906), considerada praga agrícola de relevante importância.

A precipitação foi desuniforme durante o período das coletas. A umidade oscilou durante todo o período, com picos durante o outono (Tabela 2), período de declínio populacional para a maioria das espécies.

A queda na temperatura durante a passagem dos meses de outono e inverno mostrou clara redução na diversidade de espécies e no número de indivíduos, sendo que, para a maioria das espécies, foi verificada 
Tabela 1 - Número e porcentagens de gafanhotos capturados durante os meses de coleta na Floresta Nacional de Chapecó (dezembro de 2003 a novembro de 2004).

\begin{tabular}{|c|c|c|c|c|c|c|c|c|c|c|c|c|c|c|}
\hline Período & Dez. & Jan & Fev & Mar & Abr & Mai & Jun & Jul & Ago & Set & Out & Nov & Total & $\%$ \\
\hline \multicolumn{15}{|l|}{ Espécies } \\
\hline A. gracilis & 43 & 75 & 21 & 39 & 9 & 15 & 1 & 1 & 1 & 2 & 20 & 17 & 244 & $10,49 \%$ \\
\hline M. adspersa & 3 & 12 & 37 & 17 & 47 & 22 & 13 & 9 & 1 & 1 & 30 & 11 & 203 & $8,73 \%$ \\
\hline P. graminea & 3 & 22 & 6 & 5 & 4 & 0 & 1 & 0 & 0 & 0 & 3 & 2 & 46 & $1,98 \%$ \\
\hline A. lineatus & 1 & 1 & 0 & 0 & 0 & 1 & 2 & 0 & 2 & 0 & 2 & 0 & 9 & $0,39 \%$ \\
\hline Amblytropidia sp. & 1 & 0 & 0 & 0 & 0 & 0 & 0 & 0 & 0 & 3 & 0 & 0 & 4 & $0,17 \%$ \\
\hline O. punctata & 4 & 9 & 5 & 4 & 5 & 6 & 5 & 10 & 0 & 6 & 8 & 2 & 64 & $2,75 \%$ \\
\hline P. nigromarginata & 1 & 2 & 18 & 14 & 7 & 2 & 1 & 0 & 2 & 6 & 1 & 0 & 54 & $2,32 \%$ \\
\hline R. brunneri & 11 & 41 & 11 & 10 & 13 & 9 & 3 & 2 & 0 & 0 & 14 & 1 & 115 & $4,95 \%$ \\
\hline R. pictus & 34 & 48 & 10 & 2 & 7 & 4 & 0 & 0 & 0 & 2 & 0 & 1 & 108 & $4,65 \%$ \\
\hline S. longicornis longicornis & 0 & 13 & 6 & 0 & 4 & 1 & 0 & 1 & 0 & 2 & 0 & 0 & 27 & $1,16 \%$ \\
\hline C. chacoensis & 0 & 0 & 0 & 0 & 2 & 1 & 2 & 1 & 0 & 2 & 0 & 0 & 8 & $0,34 \%$ \\
\hline Cylindrotettix sp. & 0 & 0 & 0 & 0 & 1 & 0 & 0 & 0 & 0 & 0 & 0 & 7 & 8 & $0,34 \%$ \\
\hline D. elongatus & 4 & 58 & 30 & 38 & 24 & 6 & 6 & 5 & 0 & 4 & 25 & 4 & 204 & $8,77 \%$ \\
\hline D. misionensis & 71 & 97 & 47 & 44 & 23 & 20 & 11 & 8 & 9 & 17 & 8 & 1 & 356 & $15,31 \%$ \\
\hline R. bergi & 65 & 44 & 45 & 29 & 12 & 14 & 5 & 5 & 2 & 4 & 10 & 4 & 239 & $10,28 \%$ \\
\hline S. lemniscata & 1 & 4 & 0 & 0 & 0 & 0 & 0 & 0 & 0 & 0 & 0 & 3 & 8 & $0,34 \%$ \\
\hline A. flavolineata & 1 & 0 & 0 & 0 & 0 & 0 & 3 & 0 & 2 & 15 & 29 & 4 & 54 & $2,32 \%$ \\
\hline C. speciosa & 37 & 11 & 2 & 11 & 0 & 0 & 0 & 0 & 0 & 0 & 3 & 0 & 64 & $2,75 \%$ \\
\hline S. v.viridicata & 11 & 4 & 7 & 4 & 2 & 0 & 0 & 0 & 0 & 0 & 20 & 10 & 58 & $2,49 \%$ \\
\hline$X$. discoideus discoideus & 16 & 29 & 9 & 1 & 4 & 5 & 2 & 5 & 6 & 3 & 16 & 1 & 97 & $4,17 \%$ \\
\hline Z. iheringi & 2 & 4 & 1 & 15 & 0 & 0 & 0 & 0 & 0 & 3 & 13 & 3 & 41 & $1,76 \%$ \\
\hline Z. tarsata & 2 & 1 & 0 & 0 & 1 & 0 & 0 & 0 & 0 & 0 & 0 & 0 & 4 & $0,17 \%$ \\
\hline O. virens & 13 & 62 & 41 & 33 & 27 & 0 & 0 & 6 & 0 & 1 & 2 & 4 & 189 & $8,13 \%$ \\
\hline T. subulata & 9 & 11 & 19 & 13 & 7 & 3 & 3 & 4 & 11 & 4 & 22 & 14 & 120 & $5,16 \%$ \\
\hline T. politus & 0 & 0 & 0 & 0 & 0 & 0 & 0 & 0 & 0 & 0 & 0 & 1 & 1 & $0,04 \%$ \\
\hline Total mês: & 333 & 548 & 315 & 279 & 199 & 109 & 58 & 57 & 36 & 75 & 226 & 90 & 2325 & \\
\hline Porcentagem (\%) & 14,32 & 23,57 & 13,55 & 12 & 8,56 & 4,69 & 2,49 & 2,45 & 1,55 & 3,23 & 9,72 & 3,87 & 100 & \\
\hline
\end{tabular}

queda brusca na ocorrência durante este período, aumentando novamente na primavera (Tabela1). Porém, a espécie $\boldsymbol{M}$. adspersa mostrou-se díspar em relação às demais, apresentando considerável aumento de ocorrência no outono (Tabela 1). Esta persistência à estação de clima ameno atribui-se às características mais resistentes, tendo em vista a preferência por ambientes úmidos ou até inundados, conforme DURANTON et al. (1987), e a ocorrência em locais tipicamente frios como os Andes (CARBONELL et al., 2006). A análise de regressão indicou que os índices de ocorrência de algumas espécies sofreram interferência significativa dos fatores ecológicos. Para estes foram obtidas as seguintes equações de regressão e os respectivos coeficientes de determinação $\left(\mathrm{R}^{2}\right)$ :

A. gracilis: $\mathrm{N}=1,03 \mathrm{TME}-0,43 \mathrm{TMI}-0,51 \mathrm{TMA}-$ 0,18UMD+0,35UMI-O,67UMA+0,16P; $\left(\mathrm{R}^{2}=0,97\right)$

P. graminea: $\mathrm{N}=1,36 \mathrm{TME}-0,97 \mathrm{TMI}-0,48 \mathrm{TMA}-$ $0,16 \mathrm{UMD}+0,43 \mathrm{UMI}-0,82 \mathrm{UMA}+0,01 \mathrm{P} ;\left(\mathrm{R}^{2}=0,95\right)$
R. bruneri: N=,40TME-0,77TMI-0,79TMA-0,05UMD$0,34 \mathrm{UMI}-0,78 \mathrm{UMA}+0,10 \mathrm{P}\left(\mathrm{R}^{2}=0,92\right)$

X. discoideus: N=1,38TME-1,29TMI-0,82TMA$0,47 \mathrm{UMD}+0,46 \mathrm{UMI}+0,80 \mathrm{UMA}+0,55 \mathrm{P}\left(\mathrm{R}^{2}=0,92\right)$, em que: N: Espécie, TME: Temperatura média, TMI: Temperatura mínima, TMA: Temperatura máxima, UMD: Umidade média, UMI: Umidade mínima, UMA: Umidade máxima e P: Precipitação mensal.

Os gafanhotos apresentaram variação nos índices de ocorrência ao longo do período, sendo que os picos populacionais estão diretamente relacionados com os maiores índices de temperatura.

A análise de regressão apontou a temperatura e a umidade como as variáveis climáticas que apresentam maior interferência sobre a flutuação dos gafanhotos. As espécies que são praga em potencial, apresentaram menor susceptibilidade à interferência dos fatores ambientais. 
Tabela 2 - Dados meteorológicos mensais, temperaturas $\left({ }^{\circ} \mathrm{C}\right)$ média (TME), mínima (TMI), máxima (TMA, umidade relativa do ar (UR\%) média (UME), mínima (UMI), máxima (UMA) e precipitação mensal (PREC.) para o estudo na Floresta Nacional de Chapecó (dezembro de 2003 a novembro de 2004).

\begin{tabular}{|c|c|c|c|c|c|c|c|}
\hline \multirow{2}{*}{ Meses } & \multicolumn{7}{|c|}{ - } \\
\hline & TME & TMI & TMA & UME & UMI & UMA & PREC \\
\hline Dezembro & 21,63 & 12 & 31 & 72,6 & 35 & 98 & 392,7 \\
\hline Janeiro & 23,4 & 15 & 32 & 68,88 & 40 & 93 & 150 \\
\hline Fevereiro & 22,35 & 14 & 32 & 66,87 & 31 & 98 & 115,6 \\
\hline Março & 22,53 & 14 & 34 & 63,27 & 28 & 96 & 37,5 \\
\hline Abril & 21,25 & 8,4 & 32 & 73,47 & 35 & 100 & 214,6 \\
\hline Maio & 14,03 & 4 & 24 & 85,3 & 49 & 100 & 127,9 \\
\hline Junho & 15,64 & $-1,2$ & 26 & 75,44 & 29 & 100 & 37,2 \\
\hline Julho & 13,88 & $-1,2$ & 27 & 74,34 & 35 & 100 & 177,4 \\
\hline Agosto & 16,35 & 0,2 & 29 & 65,06 & 30 & 100 & 41,7 \\
\hline Setembro & 19,67 & 8,4 & 34 & 74,57 & 35 & 99 & 251,7 \\
\hline Outubro & 19,3 & 9,6 & 28 & 63,63 & 23 & 98 & 301,4 \\
\hline Novembro & 20,55 & 11 & 32 & 68,93 & 36 & 99 & 189,9 \\
\hline
\end{tabular}

\section{REFERÊNCIAS}

CARBONELL, C.S. et al. Espécies de acridomorfos (Orthoptera) de Argentina y Uruguai/ Acridomorph (Orthoptera) species of Argentina and Uruguai. Argentina: The Orthopterists‘ Society at the Museu de La Plata, 2006. (CD ROM. Publications on Orthopteran Diversity).

COSTA-NETO, E. Insetos no cardápio. Ciência Hoje, Brasília, v.27, n.161, p.63-65, 2000.

DEBREY, L.D. et al. Rangeland grasshopper management. Agricultural Experiment Station. College of Agriculture. University of WYOMING. Grasshoppers of Wyoming and the West Home Page. June 1993. Acessado em 10 fev. 2007. On line. Disponível em: http://www.sdvc.uwyo.edu/grasshopper/ rgmanage.htm.

DURANTON, J.F. et al. Guia práctico de luta contra os gafanhotos devastadores no Brazil. Roma: FAO-CIRADPRIFAS, 1987. 343p.

EMBRAPA, Monitoramento por satélite. The grasshopper of Mato Grosso. A review and of 4 years of research 19921996. Final report for the "Environment and grasshopper plague in Brasil” project. 1996. Acessado em: 04 nov. 2007. On line. Disponível em: http://www.cnpm.embrapa.br/projects/ grshop_us/index.html
IBAMA. Floresta Nacional de Chapecó. O meio ambiente em sintonia com a conservação. Chapecó, 2003. 217p.

LUTINSKI, J.A.; GARCIA F.R.M. Análise faunística de formicidae (Hymenoptera: Apocrita) em ecossistema degradado no município de Chapecó, Santa Catarina. Biotemas, Florianópolis, v.18, p.73-86, 2005.

MEYER, J R. Orthoptera. The orthopterists' Society. North Carolina, USA: State University, 2005. Acessado em: 10 jan.2006. On line. Disponível em: http://www.cals.ncsu.edu/ couse/ent425/compendium/orthop.html

RICHMAN, D.B. et al. A manual of the grasshoppers of New Mexico Orthoptera: Acrididae and Romaleidae. Las Cruces, NM. México: New Mexico State University Cooperative Extension Service, 1993. Acessado em: 10 jan. 2007. On line. Disponível em: http://www.sidney.ars.usda.gov/ grasshopper/extrnlpg/ghwywest/ghnmtoc.htm

ROSALES, S.A. Diagnosis de Acridoideos (Orthoptera: Acridoidea) que se asocian a áreas agrícolas en la región central de México. México: Instituto de Fitosanidad del Colegio de Postgraduados Montecillo, 1996. Acessado em 06 fev.2007. On line. Disponível em: http:// www.queretaro.gob.mx/sedea/CESAVEQ/pdfs/I.pdf .

ROWELL, H. Acridoidea. 2001. Version 01 January 2001 (temporary). Acessado em 10 jan. 2007. On line. Disponível em: http://tolweb.org/Acridoidea/13290/2001.01.01 in The Tree of Life Web. 